\title{
Preface to TCHES 2018
}

\author{
Daniel Page ${ }^{1}$ and Matthieu Rivain ${ }^{2}$ \\ 1 University of Bristol, Merchant Venturers Building, \\ Woodland Road, Bristol, BS8 1UB, United Kingdom. \\ csdsp@bristol.ac.uk \\ ${ }^{2}$ CryptoExperts, 41 Boulevard des Capucines, 75002 Paris, France. \\ matthieu.rivain@cryptoexperts.com
}

Having been established in 1999, the Cryptographic Hardware and Embedded Systems (CHES) conference is today the premier venue for research on both design and analysis of cryptographic hardware and software implementations. As an area conference of the International Association for Cryptologic Research (IACR), CHES bridges the cryptographic research and engineering communities, and attracts participants from academia, industry, government and beyond.

CHES 2018 was held in Amsterdam, The Netherlands, September 9-12, 2018. It was the twentieth edition of the conference, but the first under a new hybrid (i.e., a mixture of journal and conference), gold open-access (under the Creative Commons CC-BY 4.0 license) publication model: accepted papers constituting the CHES 2018 program were published in the IACR Transactions on Cryptographic Hardware and Embedded Systems (TCHES) Volume 2018, Issues 1, 2, and 3. The decision to adopt this publication model was made by the CHES Steering Committee in early 2017. Motivated by success of other communities such as FSE, whose program is published under the same model in the IACR Transactions on Symmetric Cryptography (ToSC), it was viewed as a means of improving review and publication quality while retaining the highly successful, community-focused event, plus satisfying modern demand for (and impact of) open-access research.

The change in publication model demanded a new review process. Although the TCHES web-site houses a comprehensive FAQ detailing both, the latter can be summarised by three differences: while retaining a double-blind policy, and following IACR policy on declaration and management of Conflict of Interests (CoI), the process for each TCHES issue involves a) four submission deadlines associated with TCHES issues for a given CHES conference (only three for this first edition), b) reviewers drawn from an Editorial Board who write detailed, careful reviews and c) a richer set of possible decisions, adding journal-like major and minor revision options to outright accept or reject. The decision for each submission is informed by careful discussion between reviewers mediated by the Co-Editors-in-Chief, and a rebuttal phase allowing authors to respond to preliminary reviews. To provide the highest quality feedback to authors, significant effort is made by reviewers to update the preliminary reviews: ideally the final reviews capture discussion points, and justify the resulting decision. We expect both the publication model and review process to evolve over time, to reflect experience, and emerging challenges and opportunities. However, in our view it has already proven successful. The quantity and quality of submissions has been excellent, and, through the review quality and options for revision, we feel it has been possible to further improve both accepted and rejected submissions.

The submission statistics of TCHES Volume 2018 are summarized in Table 1. The three issues have received a total of 181 submissions (164 discounting re-submissions) among which 47 have finally been accepted, making a global acceptance rate of $26 \%$. For the re-submissions following a requested major revision, the acceptance rate soars to $60 \%$, 
Table 1: Submission statistics of TCHES Volume 2018.

\begin{tabular}{|l|c|c|c|}
\hline & Issue 1 & Issue 2 & Issue 3 \\
\hline Number of new submissions & 45 & 43 & 76 \\
\hline Number of re-submissions from previous issues & - & 5 & 12 \\
\hline Number of submissions (total) & 45 & 48 & 88 \\
\hline Number of accepted submissions & 11 & 13 & 23 \\
\hline Acceptance rate & $24 \%$ & $29 \%$ & $26 \%$ \\
\hline
\end{tabular}

which shows the effectiveness of the new process.

After voting, the Editorial Board conferred the CHES 2018 best paper award to Cold Boot Attacks on Ring and Module LWE Keys Under the NTT by Martin Albrecht, Amit Deo, and Kenneth Paterson, who were also afforded a double-length presentation slot as a result. The program included two invited talks: (Why) Are Microarchitectural Attacks really different than Physical Side-Channel Attacks? by Daniel Gruss (Graz University of Technology) and Leveraging deep-learning to perform SCA attacks against AES implementations by Elie Bursztein (Google). Following their success in previous editions, the conference also held a poster session as well as two pre-conference tutorials: Counterfeit Integrated Circuits: Threats, Detection, and Avoidance by Domenic Forte (University of Florida) and Rajat Subhra Chakraborty (Indian Institute of Technology, Kharagpur), and Formal Verification of Masked Implementations by Sonia Belaïd (CryptoExperts) and Benjamin Grégoire (INRIA). We feel all these elements reflect both hot-topics for, and the traditional influence of both academia and industry on, CHES as a whole.

Acknowledgments. Both the TCHES journal and CHES conference are the culmination of a huge, and often unseen or under-appreciated, amount of effort by a range of parties. We would like to extend our deep, sincere thanks to all of them. In particular, the conference budget was supported by the generous sponsorship of Rambus, ALPhANOV, eshard, NewAE Technology, NXP, Thales, Infineon Technologies, Robert Bosch LLC, Continental, CryptoExperts, Fox-IT, Idemia, IoTeX, OSR, PQ Shield, Secure-IC, and NSF. The conference logistics were expertly managed by the General Chairs: Ileana Buhan and Peter Schwabe. We are grateful to Sonia Belaïd for chairing the poster session, and to Daniel Genkin and Yuval Yarom for chairing the rump session. We also thank Ileana Buhan, Karim Tobich and Emmanuel Prouff for organising the CHES 2018 CTF. The schedule demanded by multiple TCHES submission deadlines, added to by workload stemming from "long" (i.e., beyond 20 pages) submissions and shepherding of those with minor revisions, has been a significant challenge. The dedication and professionalism of the Editorial Board members, and external reviewers, played a central role in successfully addressing this challenge. Instances of the websubrev software, authored and supported by Shai Halevi, enabled the TCHES submission and review processes. The publication of TCHES was supported by Tim Güneysu in the role of Managing Editor; constituent papers all used a $\mathrm{LT}_{\mathrm{E} X} \mathrm{X}$ style originally authored by Gaëtan Leurent. We are indebted to the CHES Steering Committee for advice and support throughout the development of and transision to the new publication model. We capitalised on experience from various members of the FSE/ToSC community, and we are gratefull to the FSE Steering Committee for time and advice. Last but not least, we would like to express our deep gratitude to the authors of all the submissions for their vital contribution to the success of this first edition of the (T)CHES journal/conference hybrid. 


\section{Editorial Board}

Diego Aranha

Roberto Avanzi

Lejla Batina

Sonia Belaïd

Daniel J. Bernstein

Joppe W. Bos

Billy Brumley

Ileana Buhan

Chen-Mou Cheng

Christophe Clavier

Thomas Eisenbarth

Junfeng Fan

Sebastian Faust

Viktor Fischer

Wieland Fischer

Pierre-Alain Fouque

Jacques Fournier

Georges Gagnerot

Benedikt Gierlichs

Aline Gouget

Jorge Guajardo

Shay Gueron

Tim Güneysu

Mike Hamburg

Annelie Heuser

Naofumi Homma

Kimmo Järvinen

Marc Joye

Emilia Käsper

Kerstin Lemke-Rust

Tancrède Lepoint

Patrick Longa

Roel Maes

Stefan Mangard

Clémentine Maurice

Atsuko Miyaji

Amir Moradi

Debdeep Mukhopadhyay

Colin O'Flynn

Elisabeth Oswald

Daniel Page

Thomas Peyrin

Axel Poschmann

Emmanuel Prouff

Francesco Regazzoni

Matthieu Rivain

Erkay Savaş

Peter Schwabe

Sergei Skorobogatov

François-Xavier Standaert

Ruggero Susella

Mike Tunstall

Srinivas Vivek

Bo-Yin Yang

Yuval Yarom

Rina Zeitoun
University of Campinas, Brazil

ARM, Germany

Radboud University, The Netherlands

CryptoExperts, France

University of Illinois at Chicago, USA

NXP Semiconductors, Belgium

Tampere University of Technology, Finland

Riscure, The Netherlands

National Taiwan University, Taiwan

Université de Limoges, France

University of Lübeck \& WPI, Germany

Open Security Research Inc., China

TU Darmstadt, Germany

Jean Monnet University, Saint-Etienne, France

Infineon Technologies, Germany

Université Rennes 1, France

CEA-Leti, France

eshard, France

KU Leuven, Belgium

Gemalto, France

Robert Bosch LLC - Research and Technology Center, USA

Amazon Web Services \& University of Haifa, Israel

Ruhr University Bochum \& DFKI, Germany

Rambus, USA

CNRS, IRISA, France

RIEC/Tohoku University, Japan

University of Helsinki, Finland

NXP Semiconductors, USA

Google, USA

Bonn-Rhein-Sieg University of Applied Sciences, Germany

SRI International, USA

Microsoft Research, USA

Intrinsic ID, The Netherlands

TU Graz, Austria

CNRS, IRISA, France

Osaka Univirsity/JAIST, Japan

Ruhr University Bochum, Germany

Indian Institute of Technology Kharagpur, India

NewAE Technology Inc., Canada

University of Bristol, United Kindom

University of Bristol, United Kingdom - Co-Editor-in-Chief

Nanyang Technological University, Singapore

DarkMatter LLC, United Arab Emirates

ANSSI, France

ALaRI - USI, Switzerland

CryptoExperts, France - Co-Editor-in-Chief

Sabanci University, Turkey

Radboud University, The Netherlands

University of Cambridge, United Kindom

Universite catholique de Louvain, Belgium

STMicroelectronics, Italy

Rambus, USA

IIIT Bangalore, India

Academia Sinica, Taiwan

University of Adelaide \& Data61, Australia

IDEMIA, France 


\section{External Reviewers}

\begin{tabular}{|c|c|c|}
\hline Alexandre Adomnicai & Daniele Grattarola & Romain Poussier \\
\hline Anita Aghaie & Aurélien Greuet & Robert Primas \\
\hline Estuardo Alpírez Bock & Hannes Gross & Jürgen Pulkus \\
\hline Pedro G. M. R. Alves & Vincent Grosso & Thomas Pöppelmann \\
\hline Christopher Ambrose & Berk Gulmezoglu & Shahram Rasoolzadeh \\
\hline Florian Bache & Patrick Haddad & Joost Renes \\
\hline Anubhab Baksi & Carl-Daniel Hailfinger & Oscar Reparaz \\
\hline Valentina Banciu & Sohaib ul Hassan & Léo Reynaud \\
\hline Guillaume Barbu & Matthias Hiller & Jefferson Ricardini \\
\hline Alberto Battistello & Kristina Hostakova & Bastian Richter \\
\hline Pierre Bayon & Yuan-Che Hsu & Franck Rondepierre \\
\hline Emanuele Bellini & Andreas Hülsing & Debapriya Basu Roy \\
\hline Luk Bettale & Christopher Huth & Sujoy Sinha Roy \\
\hline Shivam Bhasin & Michael Hutter & Vladimir Rožić \\
\hline Sarani Bhattacharya & Gorka Irazoqui & Markku-Juhani O. Saarinen \\
\hline Elif Bilge Kavun & Takanori Isobe & Simona Samardjiska \\
\hline Begül Bilgin & Mustafa Kairallah & Peter Samarin \\
\hline Nina Bindel & Miroslav Knezevic & Falk Schellenberg \\
\hline Manuel Bluhm & Lieneke Kusters & Werner Schindler \\
\hline Matteo Bocchi & Maxime Lecomte & Tobias Schneider \\
\hline Jakub Breier & Antoine Loiseau & Okan Seker \\
\hline Ahmet Can Mert & David Lubicz & Mitsuru Shiozaki \\
\hline Anupam Chattopadhyay & Pedro Maat C. Massolino & Chunhua $\mathrm{Su}$ \\
\hline Wenjie Che & Houssem Maghrebi & Banik Subhadeep \\
\hline Yu-Chia Chen & Mark Marson & Robert Szerwinski \\
\hline Lukasz Chmielewski & Dan Martin & Yannick Teglia \\
\hline Tung Chou & Silvia Mella & Hugues Thiebeauld \\
\hline Jessy Clediere & Vincent Migliore & Adrian Thillard \\
\hline Brice Colombier & Ahmad Moghimi & Karim Tobich \\
\hline Jean-Sébastien Coron & Thorben Moos & Harshal Tupsamudre \\
\hline Joan Daemen & Andres Moreno & Nicola Tuveri \\
\hline Nilanjan Datta & Ugo Mureddu & Rei Ueno \\
\hline Elke De Mulder & Michael Naehrig & Markus Ullmann \\
\hline Nicolas Debande & Zakaria Najm & Dan Ungureanu \\
\hline Thomas Decnudde & Christophe Negre & Thomas Unterluggauer \\
\hline Jeroen Delvaux & Ventzi Nikov & Praveen Vadnala \\
\hline Markus Dichtl & Elie Noumon Allini & Aurelien Vasselle \\
\hline Cécile Dumas & Tobias Oder & Vincent Verneuil \\
\hline Baris Ege & Shinya Okumura & Pim Vullers \\
\hline Maria Eichlseder & Erdinc Ozturk & Junwei Wang \\
\hline Nadia El Mrabet & Clara Paglialonga & Felix Wegener \\
\hline Maik Ender & Kostas Papagiannopoulos & Mario Werner \\
\hline Thomas Espitau & Sikhar Patranabis & Carolyn Whitnall \\
\hline Benoit Feix & Florian Pebay Peyroula & Alexander Wild \\
\hline Alberto Ferrante & Cesar Pereida García & Antoine Wurcker \\
\hline Si Gao & Peter Pessl & Shayan Yassami \\
\hline Santosh Ghosh & Stjepan Picek & Ville Yli-Mäyry \\
\hline Gilbert Goodwill & Christian Pilato & Shih-Chun You \\
\hline Dahmun Goudarzi & Jim Plusquellic & Daniele Zambon \\
\hline
\end{tabular}

